\title{
The Fifth Maximum Wiener Index of Uniform Hypergraphs
}

\author{
Yalan $\mathrm{Li}^{1}$, Bo Deng ${ }^{2,3}$, Chengfu Ye ${ }^{2}$, Feng Fu ${ }^{2}$, Huilong Chen ${ }^{2}$ \\ ${ }^{1}$ School of Computer, Qinghai Normal University, Xining, China \\ ${ }^{2}$ School of Mathematics and Statistics, Qinghai Normal University, Xining, China \\ ${ }^{3}$ College of Science, Guangdong University of Petrochemical Technology, Maoming, China
}

\section{Email address:}

liyalan2017@163.com (Yalan Li),dengbo450@163.com (Bo Deng), yechf@qhnu.edu.cn (Chengfu Ye)

\section{To cite this article:}

Yalan Li, Bo Deng, Chengfu Ye, Feng Fu, Huilong Chen. The Fifth Maximum Wiener Index of Uniform Hypergraphs. American Journal of Mathematical and Computer Modelling. Vol. 4, No. 3, 2019, pp. 74-82. doi: 10.11648/j.ajmcm.20190403.14

Received: August 1, 2019; Accepted: August 23, 2019; Published: September 10, 2019

\begin{abstract}
Hypergraph theory has been found many applications in chemistry. As an important descriptor of molecular structures, the Wiener index of a graph also has many applications. The Wiener index of a connected hypergraph is defined as the summation of distances between all pairs of vertices. If each edge contains exactly k vertices, then a hypergraph $\mathrm{G}$ is called k-uniform. A hypertree is a connected hypergraph with no cycles. For k-uniform hypertree, H. Guo, B. Zhou et al. have determined the first, second and third maximum and minimum Wiener indices of uniform hypertrees. And give the unique structure of the k-uniform hypertree corresponding to the Wiener index, Moreover, in this paper, We first find out the relationship between the first few Wiener indices, then according to the structure of the graph, determine the unique k-uniform hypertree with the fifth maximum Wiener index. Through the determination of the fifth Wienr index k-uniform hypertree, the structure of the NTH Wiener index k-uniform hypertree can be found.
\end{abstract}

Keywords: Wiener Index, K-uniform Hypertree, The Fifth Maximum

\section{Introduction}

Let $V(G)$ and $E(G)$ be the vertex a hypergraph $G$ is called $k$-uniform. When $k=2$, an ordinary graph $G$ is a 2 uniform hypergraph. A hypertree is a connected hypergraph with no cycles. A $k$-uniform hypertree with $m$ edges always has $1+(k-1) m$ vertices. The degree of a vertex $v \in V(G)$, denoted by $d_{G}(v)$, is the number of edges of $G$ which contains the vertex $v[1]$.

For $u, v \in V(G)$, a path from $u$ to $v$ in $G$ is defined to be a sequence of vertices and edges $\left(v_{0}, e_{1}, v_{1}, \ldots, v_{p-1}, e_{p}, v_{p}\right)$ with all $v_{i}$ s distinct and $e_{i}$ s distinct such that $v_{i-1}, v_{i} \in e_{i}$ for $i=1,2, \ldots p$, where $v_{0}=u$ and $v_{p}=v$. A cycle in $G$ is a sequence of vertices and edges $\left(v_{0}, e_{1}, v_{1}, \ldots, v_{p-1}, e_{p}, v_{p}\right)$ with $p \geq 2$, all $v_{i} \mathrm{~s}$ distinct except $v_{0}=v_{p}$ and all $e_{i} \mathrm{~s}$ distinct such that $v_{i-1}, v_{i} \in e_{i}$ for $i=1,2, \ldots p$, where the value $p$ is the length of this path or cycle. For any $u, v \in V(G)$, if it exits a path from $u$ to $v$ for any $u, v \in V(G)$, then $G$ is called connected. Let $G$ be a $k$-uniform hypergraph with
$V(G)=\left\{v_{1}, v_{2}, \ldots, v_{n}\right\}$. For $u, v \in V(G)$, the distance between $u$ and $v$ in $G$ is denoted by $d_{G}(u, u)$. In particular, $d_{G}(u, u)=0$. The diameter of $G$ is the maximum distance among all vertex pairs of $G[1]$.

Hypergraph theory has been found many applications in chemistry [2-4]. The reseach in the study [3] indicated that the hypergraph model shows a higher accuracy of molecular structure. That is the higher accuracy of the model and the greater diversity of the behavior of its invariants. As an important descriptor of molecular structures, the Wiener index of a graph also has many applications [2-5].

The Wiener index $W(G)$ of $G$ is defined as the summation of distances among all unordered pairs of distinct vertices $u, v$ in $G$, i.e., $W(G)=\sum_{\{u, v\} \subseteq V(G)} d_{G}(u, v)$. Especially, the summation of distances from the vertex $u$ to any other vertex, is denoted by $W_{G}(u)=\sum_{v \in V(G)} d_{G}(u, v)$. Obviously, we see that $W(G)=\frac{1}{2} W_{G}(u)$. H. Guo et al. [4] have determined the 
first, second and third maximum and minimum Wiener indices of uniform hypertrees.

Moreover, in this paper, We determine the unique $k$ uniform hypertree with the fifth maximum Wiener index.

\section{Preliminary}

Let $G$ be a connected $k$-uniform hypergraph with $u, v \in e \in E(G)$. For nonnegative integers $p$ and $q$, let $G_{u, v}(p, q)$ be the $k$-uniform hypergraph obtained from $G$ by attaching a pendant path of length $p$ at $u$ and a pendant path of length $q$ at $v$ [4].

Proposition 2.1 [4]. Let $G$ be a connected $k$-uniform hypergraph with $|E(G)| \geq 2, u, v \in e$ and $d_{G}(u)=1$. For integers $p \geq q \geq 1, W\left(G_{u, v}(p, q)\right)<W\left(G_{u, v}(p+1, q-1)\right.$.

For positive integers $p$ and $q$, and a $k$-uniform hypergraph $G$, let $G_{u}(p, q)$ be the $k$-uniform hypergraph obtained from $G$ by attaching two pendant paths of length $p$ and $q$ at $u$, respectively, and $G_{u}(p, 0)$ be the $k$-uniform hypergraph obtained from $G$ by attaching a pendant paths of length $p$ at $u$.

Proposition 2.2 [4]. Let $G$ be a connected $k$-uniform hypergraph with $|E(G)| \geq 1$ and $u \in V(G)$. For integers $p \geq q \geq 1, W\left(G_{u}(p, q)\right)<W\left(G_{u}(p+1, q-1)\right.$.

For a $k$-uniform hypertree $G$ with $V(G)=\left\{v_{1}, v_{2}, \ldots, v_{n}\right\}$, if $E(G)=\left\{e_{1}, e_{2}, \ldots, e_{n}\right\}$, where $e_{i}=\left\{v_{(i-1)(k-1)+1}, \ldots, v_{(i-1)(k-1)+k}\right\}$ for $i=1,2, \ldots m$, then we call $G$ a $k$-uniform loose path, denoted by $p_{n, k}$.

For a $k$-uniform hypertree $G$ of order $n$, if there is a disjoint partition of the vertex set $V(G)=\{u\} \cup V_{1} \cup \ldots \cup V_{m}$ such that $\left|V_{1}\right|=\ldots=\left|V_{m}\right|=k-1$, and $E(G)=\left\{\{u\} \cup V_{i}: 1 \leq i \leq m\right\}$, then we call $G$ is a $k$-uniform hyperstar (with center $u$ ), denoted by $S_{n, k}$. In particular, $S_{1, k}$ is a hypergraph with a single vertex and $S_{k, k}$ is a hypergraph with a single edge.

For positive integers $\Delta, n$ with $1 \leq \Delta \leq \frac{n-1}{k-1}$, let $B_{n, k}^{\Delta}$ be the $k$-uniform hypertree obtained from vertex-disjoint hyperstar $S_{(\Delta-1)(k-1)+1, k}$ with center $u$ and loose path $P_{n-(\Delta-1)(k-1), k}$ with an end vertex $v$ by identifying $u$ and $v$. In particular, $B_{n, k}^{\Delta} \cong P_{n, k}$ if $\Delta=1,2$.

\section{Hypertree with the Fifth Maximum Wiener Index}

In this section, we determine the unique $k$-uniform hypertree with the fifth maximum Wiener index.

Theorem 3.1 [5]. Let $T$ be a $k$-uniform hypertree on $n$ vertices with maximum degree $\Delta$, where $1 \leq \Delta \leq \frac{n-1}{k-1}$. Then $W(T) \leq W\left(B_{n, k}^{\Delta}\right)$ with equality if and only if $T \cong B_{n, k}^{\Delta}$.
For $k \geq 3, \quad \frac{\mathrm{n}-1}{k-1} \geq 3$ and a loose path $P_{n-k+1, k}=\left(u_{0}, e_{1}, u_{1}, \ldots, e_{\frac{n-k}{k-1}}, u_{\frac{n-k}{k-1}}\right)$, let $F_{n, k}$ be the $k$-uniform hypertree obtained from $P_{n-k+1, k}$ by attaching a pendant edge at a vertex in $e_{2} \backslash\left\{u_{1}, u_{2}\right\}$. If $\frac{n-1}{k-1}=3$, then $F_{n, k} \cong P_{n, k}$. Let $F_{n, 2}=B_{n, 2}^{3}$.

Theorem 3.2 [5]. For $\frac{n-1}{k-1} \geq 1$. Let $T$ be a $k$-uniform hypertree on $n$ vertices. Then $W(T) \leq W\left(P_{n, k}\right)$ with equality if and only if $T \cong P_{n, k}$.

Lemma 3.1 [5]. Suppose that $k \geq 3$ and $\frac{n-1}{k-1} \geq 3$. Then $W\left(B_{n, k}^{3}\right)<W\left(F_{n, k}\right)$.

For $k \geq 3, \frac{n-1}{k-1} \geq 6$ and a loose path $P_{n-k+1, k}=\left(u_{0}, e_{1}, u_{1}, \ldots, e_{\frac{n-k}{k-1}}, u_{\frac{n-k}{k-1}}\right)$, let $E_{n, k}$ be the $k$-uniform hypertree obtained from $P_{n-k+1, k}$ by attaching a pendant edge at a vertex in $e_{3} \backslash\left\{u_{2}, u_{3}\right\}$. Let $E_{n, 2}$ be the $k$-uniform hypertree obtained from $P_{n-k+1, k}$ by attaching a pendant edge at $u_{2}$.

Theorem 3.3 [4]. For $\frac{n-1}{k-1} \geq 4$, let $T$ be a $k$-uniform hypertree with $n$ vertices. Suppose that $T \neq P_{n, k}$. Then $W(T) \leq W\left(F_{n, k}\right)$ with equality if and only if $T \cong F_{n, k}$.

Lemma 3.2 [4]. Suppose that $k \geq 3$ and $\frac{n-1}{k-1} \geq 6$. Then $W\left(B_{n, k}^{3}\right) \geq W\left(E_{n, k}\right)$ with equality if and only if $\frac{n-1}{k-1}=6$.

Lemma 3.3 For $k \geq 3,3 \leq i \leq \frac{n-1}{k-1}-3, \frac{n-1}{k-1} \geq 6$ and a loose path $P_{n-k+1, k}=\left(u_{0}, e_{1}, u_{1}, \ldots, e_{\frac{n-k}{k-1}}, u_{\frac{n-k}{k-1}}\right)$, let $H_{n, k}^{i}$ be the $k-$ uniform hypertree obtained from $P_{n-k+1, k}$ by attaching a pendant edge at a vertex in $e_{i} \backslash\left\{u_{i-1}, u_{i}\right\}$. Then $W\left(B_{n, k}^{3}\right) \geq W\left(H_{n, k}^{i}\right)$ with equality if and only if $i=3$ and $\frac{n-1}{k-1}=6$.

Proof. If $i=3, H_{n, k}^{3}=E_{n, k}$, by Lemma 3.2, we have $W\left(B_{n, k}^{3}\right) \geq W\left(E_{n, k}\right)$ with equality if and only if $\frac{n-1}{k-1}=6$. Suppose $4 \leq i \leq \frac{n-1}{k-1}-3$, let $T=H_{n, k}^{i}, v \in e_{i} \backslash\left\{u_{i-1}, u_{i}\right\}$, with $d_{T}(v)=2$, and let e be the pendant edge at $v$ in $T$. Let $T^{\prime}$ be the hypergraph obtained from $T$ by moving $e$ from $v$ to $u_{1}$, obviously, $T^{\prime} \cong B_{n, k}^{3}$, Let $V_{1}=V(T) \backslash(e \backslash\{v\})$, Note that

$$
W_{T}\left(V_{1}\right)=W_{T^{\prime}}\left(V_{1}\right) \quad, \quad W_{T}(e \backslash\{v\})=W_{T^{\prime}}(e \backslash\{v\})
$$


$W_{T}\left(e \backslash\{v\}, e_{2} \cup e_{3} \cup \cdots \cup e_{i}\right)=W_{T^{\prime}}\left(e \backslash\{v\}, e_{2} \cup e_{3} \cup \cdots \cup e_{i}\right)$.

From $T$ to $T^{\prime}$, the distance between a vertex of $e \backslash\{v\}$ and a vertex of $e_{1} \backslash\left\{u_{1}\right\}$ decreases by $i-1$, and the distance between a vertex of $e \backslash\{v\}$ and a vertex of

Then

$$
W_{T}\left(e \backslash\{v\}, e_{1} \backslash\left\{u_{1}\right\}\right)-W_{T^{\prime}}\left(e \backslash\{v\}, e_{1} \backslash\left\{u_{1}\right\}\right)=(i-1)|e \backslash\{v\}|\left|e_{1} \backslash\left\{u_{1}\right\}\right|=(i-1)(k-1)^{2}
$$

and

$$
\begin{aligned}
& W_{T}\left(e \backslash\{v\}, V_{1} \backslash\left(e_{1} \cup e_{2} \cup \cdots \cup e_{i}\right)\right)-W_{T^{\prime}}\left(e \backslash\{v\}, V_{1} \backslash\left(e_{1} \cup e_{2} \cup \cdots \cup e_{i}\right)\right) \\
& =-(i-2)\left(\frac{n-1}{k-1}-i-1\right)(k-1)^{2} .
\end{aligned}
$$

Since

$$
\begin{aligned}
& W(T)=W_{T}\left(V_{1}\right)+W_{T}(e \backslash\{v\})+W_{T}\left(e \backslash\{v\}, e_{2} \cup e_{3} \cup \cdots \cup e_{i}\right) \\
& +W_{T}\left(e \backslash\{v\}, e_{1} \backslash\left\{u_{1}\right\}\right)+W_{T}\left(e \backslash\{v\}, V_{1} \backslash\left(e_{1} \cup e_{2} \cup \cdots \cup e_{i}\right)\right) .
\end{aligned}
$$

and

$$
W\left(T^{\prime}\right)=W_{T^{\prime}}\left(V_{1}\right)+W_{T^{\prime}}\left(e \backslash\{v\}, e_{2} \cup e_{3} \cup \cdots \cup e_{i}\right)+W_{T^{\prime}}\left(e \backslash\{v\}, e_{1} \backslash\left\{u_{1}\right\}\right)+W_{T^{\prime}}\left(e \backslash\{v\}, V_{1} \backslash\left(e_{1} \cup e_{2} \cup \cdots \cup e_{i}\right)\right) .
$$

We have

$$
\begin{aligned}
& W(T)-W\left(T^{\prime}\right)=W_{T}\left(e \backslash\{v\}, e_{1} \backslash\left\{u_{1}\right\}\right)+W_{T}\left(e \backslash\{v\},\left(e_{1} \cup e_{2} \cup \cdots \cup e_{i}\right)\right)-W_{T^{\prime}}\left(e \backslash\{v\}, e_{1} \backslash\left\{u_{1}\right\}\right)-W_{T^{\prime}}\left(e \backslash\{v\}, V_{1} \backslash\left(e_{1} \cup e_{2} \cup \cdots \cup e_{i}\right)\right) \\
& =(i-1)(k-1)^{2}-(i-2)\left(\frac{n-1}{k-1}-i-1\right)(k-1)^{2} \\
& =\left[(i-1)-(i-2)\left(\frac{n-1}{k-1}-i-1\right)\right](k-1)^{2} \leq 0 .
\end{aligned}
$$

Then we can see that $W\left(B_{n, k}^{3}\right) \geq W\left(H_{n, k}^{i}\right)$, for $3 \leq i \leq \frac{n-1}{k-1}-3$, with equality holds if and only if $i=3$ and $\frac{n-1}{k-1}-6$

For $\quad k \geq 3, \frac{n-1}{k-1} \geq 8 \quad$ and $\quad$ a loose path $P_{n-k+1, k}=\left(u_{0}, e_{1}, u_{1}, \cdots, e_{\frac{n-k}{k-1}}, u_{\frac{n-k}{k-1}}\right), H_{n . k}^{4}$ be the k-uniform hypertree obtained from $P_{n-k+1, k}$ by attaching a pendent edge at a vertex in $e_{4} \backslash\left\{u_{3}, u_{4}\right\}$. By Lemma 3.3, we have $W\left(B_{n, k}^{3}\right) \geq W\left(H_{n, k}^{4}\right)$.

For $k=2$, let $F_{n, 2}^{\prime}$ be the tree obtained by attaching a pendent edge at the vertex $v_{3}$ of the path $v_{1}, \cdots, v_{n-1}$. For $k \geq 3$, let $F_{n, k}^{\prime}$ be the k-uniform hypertree obtained from $P_{n-k+1, k}=\left(u_{0}, e_{1}, u_{1}, \cdots, e_{\frac{n-k}{k-1}}, u_{\frac{n-k}{k-1}}\right)$ by attaching two pendant edges at $v_{1}$ and $v_{n-4}$ of the path $v_{1}, \cdots, v_{n-4}$. Let $E_{n, 2}^{\prime}$ be the tree obtained by attaching a pendant edges at the vertex $v_{4}$ of the path $v_{1}, \cdots, v_{n-1}$.
Lemma 3.4 For $k=2, n \geq 6, \Delta(T)=3 . \quad T$ be an any 2uniform hypertree obtained by attaching two pendent edges at two internal vertices of a path on $n-2$ vertices, respectively. Then $W(T) \leq W\left(F_{n, 2}^{*}\right)$ with equality if and only if $T \cong F_{n, 2}^{*}$. Proof. Let $T$ be such a hypertree with the maximum Wiener index. As $\Delta(T)=3, T$ can not be a path. Give a path $P=v_{1}, e_{1}, v_{2}, \cdots, e_{n-3}, v_{n-2}$, add the first pendent edge $e_{n-2}$ to $P$ and get a graph $T_{1}$. By Theorem 3.3, $W\left(T_{1}\right) \leq W\left(F_{n-1, k}\right)$, the edge $e_{n-2}$ must be attached at the vertex $v_{2}$. Then add the second pendant edge $e_{n-1}$ to $P$. Assume $e_{n-1}$ is attached at the vertex $v_{j}(3 \leq j \leq n-3)$ of $P$. Note that $e_{n-1}=\left\{v_{j} v_{n}\right\}$. Obviously, $T$ is composed of $T_{1}$ and $e_{n-1}$ and $W(T)=W\left(T_{1}\right)+W\left(v_{n}, T_{1}\right)$. In order to get the Wiener index of $T$, we need to calculate the maximum value of $W\left(v_{n}, T_{1}\right)$. Since

$$
\begin{aligned}
& W\left(v_{n}, T_{1}\right)=1+2+\cdots+j+j+2+3+\cdots+n-j+1 \\
& =\frac{j(j+1)}{2}+j+\frac{(n-j)(1+n-j)}{2} \\
& =\frac{2 j^{2}+(2-2 n) j+n^{2}+n}{2} .
\end{aligned}
$$


Let $f(j)=2 j^{2}+(2-2 n) j+n^{2}+n$. We can see that the function $f(j)$ attains the maximum value as $j=n-3$ so that $W(T)$ have maximum value in this case. Thus, the edge $e_{n-1}$ must be attached at the vertex $v_{n-3}$ of $P$ and $T \cong F_{n, 2}^{*}$.

Lemma 3.5 For $k \geq 3$ and $\frac{n-1}{k-1} \geq 8, W\left(E_{n, k}\right)>W\left(H_{n, k}^{4}\right)$ holds.

Proof. Note that $E_{n, k}$ be a k-uniform hypertree obtained

$$
\begin{aligned}
& W\left(T \backslash\left(e_{\frac{n-1}{k-1}} \backslash\{v\}\right)\right)=W\left(T^{\prime} \backslash\left(e_{\frac{n-1}{k-1}} \backslash\{u\}\right)\right) \\
& W_{T}\left(e_{\frac{n-1}{k-1}} \backslash\{v\}\right)=W_{T^{\prime}}\left(e_{\frac{n-1}{k-1}} \backslash\{u\}\right) \\
& W_{T}\left(e_{\frac{n-1}{k-1}} \backslash\{v\}, e_{3} \cup e_{4}\right)=W_{T^{\prime}}\left(e_{\frac{n-1}{k-1}} \backslash\{u\}, e_{3} \cup e_{4}\right) \\
& W_{T}\left(e_{\frac{n-1}{k-1}} \backslash\{v\}, e_{1} \cup\left(e_{2} \backslash\left\{u_{2}\right\}\right) \cup\left(e_{5} \backslash\left\{u_{4}\right\}\right) \cup e_{6} \cup e_{7} \cup \cdots \cup e_{\frac{n-1}{k-1}}\right) \\
& =\left(3+4+4+5+6+\cdots+\frac{n-k}{k-1}-1\right)(k-1)^{2} \\
& W_{T^{\prime}}\left(e_{\frac{n-1}{k-1}} \backslash\{u\}, e_{1} \cup\left(e_{2} \backslash\left\{u_{2}\right\}\right) \cup\left(e_{5} \backslash\left\{u_{4}\right\}\right) \cup e_{6} \cup e_{7} \cup \cdots \cup e_{\frac{n-1}{k-1}}\right) \\
& =\left(4+5+3+4+6+\cdots+\frac{n-k}{k-1}-2\right)(k-1)^{2} .
\end{aligned}
$$

Thus,

$$
\begin{gathered}
W(T)-W\left(T^{\prime}\right)=W_{T}\left(e_{\frac{n-1}{k-1}} \backslash\{v\}, e_{1} \cup\left(e_{2} \backslash\left\{u_{2}\right\}\right) \cup\left(e_{5} \backslash\left\{u_{4}\right\}\right) \cup e_{6} \cup e_{7} \cup \cdots \cup e_{\frac{n-1}{k-1}}\right) \\
-W_{T^{\prime}}\left(e_{\frac{n-1}{k-1}} \backslash\{u\}, e_{1} \cup\left(e_{2} \backslash\left\{u_{2}\right\}\right) \cup\left(e_{5} \backslash\left\{u_{4}\right\}\right) \cup e_{6} \cup e_{7} \cup \cdots \cup e_{\frac{n-1}{k-1}}\right) \\
=\left(\frac{n-1}{k-1}-7\right)(k-1)^{2}>0
\end{gathered}
$$

Then we can see that $W\left(E_{n, k}\right)>W\left(H_{n, k}^{4}\right)$ for $k \geq 3$ and $\frac{n-1}{k-1} \geq 8$.

Lemma 3.6 Suppose that $k \geq 3$ and $\frac{n-1}{k-1} \geq 4$. Then $W\left(B_{n, k}^{4}\right)<W\left(F_{n, k}^{\prime}\right)$.

Proof. Note that $F_{n, k}^{\prime}$ be the k-uniform hypertree obtained from $P_{n-k+1, k}=\left(u_{0}, e_{1}, u_{1}, \cdots, e_{\frac{n-k}{k-1}}, u_{\frac{n-k}{k-1}}\right)$ by attaching a pendent edge $\frac{e_{n-1}}{k-1}$ at a vertex in $u_{2}$. Let $T=F_{n, k}^{\prime}$. Then by moving $e_{1}$ from $u_{1}$ to $u_{2}$ in $T$, we get a hypertree $T^{\prime}$. Thus, $T^{\prime} \cong B_{n, k}^{4}$. Let $V_{1}=V(T) \backslash\left(e_{1} \backslash\left\{u_{1}\right\}\right)$. Note that

$$
\begin{gathered}
W_{T}\left(V_{1}\right)=W_{T^{\prime}}\left(V_{1}\right) \\
W_{T}\left(e_{1} \backslash\left\{u_{1}\right\}\right)=W_{T^{\prime}}\left(e_{1} \backslash\left\{u_{1}\right\}\right) \\
W_{T}\left(e_{1} \backslash\left\{u_{1}\right\}, e_{\frac{n-1}{k-1}} \backslash\left\{u_{2}\right\}\right)=3(k-1)^{2}
\end{gathered}
$$




$$
\begin{gathered}
W_{T^{\prime}}\left(e_{1} \backslash\left\{u_{1}\right\}, e_{\frac{n-1}{k-1}} \backslash\left\{u_{2}\right\}\right)=2(k-1)^{2} \\
W_{T}\left(e_{1} \backslash\left\{u_{1}\right\}, e_{2} \cup e_{3} \cup \cdots \cup e_{\frac{n-k}{k-1}}\right)=\left(2+3+4+\cdots+\frac{n-k}{k-1}\right)(k-1)^{2} \\
W_{T^{\prime}}\left(e_{1} \backslash\left\{u_{1}\right\}, e_{2} \cup e_{3} \cup \cdots \cup e_{\frac{n-k}{k-1}}\right)=\left(2+2+3+4+\cdots+\frac{n-k}{k-1}-1\right)(k-1)^{2}
\end{gathered}
$$

Since

$$
\begin{aligned}
& W(T)=W_{T}\left(V_{1}\right)+W_{T}\left(e_{1} \backslash\left\{u_{1}\right\}\right)+W_{T}\left(e_{1} \backslash\left\{u_{1}\right\}, e_{\frac{n-1}{k-1}} \backslash\left\{u_{2}\right\}\right) \\
& +W_{T}\left(e_{1} \backslash\left\{u_{1}\right\}, e_{2} \cup e_{3} \cup \cdots \cup e_{\frac{n-k}{k-1}}\right) .
\end{aligned}
$$

and

$$
\begin{aligned}
& W\left(T^{\prime}\right)=W_{T^{\prime}}\left(V_{1}\right)+W_{T^{\prime}}\left(e_{1} \backslash\left\{u_{1}\right\}\right)+W_{T^{\prime}}\left(e_{1} \backslash\left\{u_{1}\right\}, e_{\frac{n-1}{k-1}} \backslash\left\{u_{2}\right\}\right) \\
& +W_{T^{\prime}}\left(e_{1} \backslash\left\{u_{1}\right\}, e_{2} \cup e_{3} \cup \cdots \cup e_{\frac{n-k}{k-1}}\right) .
\end{aligned}
$$

We have

$$
\begin{gathered}
W(T)-W\left(T^{\prime}\right)=W_{T}\left(e_{1} \backslash\left\{u_{1}\right\}, e_{\frac{n-1}{k-1}} \backslash\left\{u_{2}\right\}\right)+W_{T}\left(e_{1} \backslash\left\{u_{1}\right\}, e_{2} \cup e_{3} \cup \cdots \cup e_{\frac{n-k}{k-1}}\right) \\
-W_{T^{\prime}}\left(e_{1} \backslash\left\{u_{1}\right\}, e_{\frac{n-1}{k-1}} \backslash\left\{u_{2}\right\}\right)-W_{T^{\prime}}\left(e_{1} \backslash\left\{u_{1}\right\}, e_{2} \cup e_{3} \cup \cdots \cup e_{\frac{n-k}{k-1}}\right) \\
=(k-1)^{2}+\left(\frac{n-k}{k-1}-2\right)(k-1)^{2} \\
=\left(\frac{n-k}{k-1}-1\right)(k-1)^{2}>0
\end{gathered}
$$

Then we can see that $W\left(F_{n, k}^{\prime}\right)<W\left(B_{n, k}^{4}\right)$ for $k \geq 3$ and $\frac{n-1}{k-1} \geq 4$.

Theorem 3.4. For $\frac{n-1}{k-1} \geq 8$, let $T$ be an any $k-$ uniform hypertree on $n$ vertices. For $k=2, T \neq F_{n, 2}^{\prime}, B_{n, 2}^{3}, P_{n, 2}$. For $k \geq 3, T \neq E_{n, k}, B_{n, 2}^{3}, F_{n, k}, P_{n, k}$. Then

(i) If $k=2$, then $W(T) \leq W\left(B_{n, 2}^{4}\right)$ or $W(T) \leq W\left(E_{n, 2}^{\prime}\right)$, with equality if and only if $T \cong B_{n, 2}^{4}$ or $T \cong E_{n, 2}^{\prime}$;

(ii) If $k \geq 3$ and $\frac{n-1}{k-1}=8$, then $W(T) \leq W\left(H_{n, k}^{4}\right)$ with equality if and only if $T \cong H_{n, k}^{4}$, and $W\left(H_{n, k}^{4}\right) \leq W\left(F_{n, k}{ }^{\prime}\right)$;

(iii) If $k \geq 3$ and $\frac{n-1}{k-1} \geq 9$, then $W(T) \leq W\left(F_{n, k}^{\prime}\right)$ with equality if and only if $T \cong F_{n, k}^{\prime}$.

Proof. Let $T$ be a hypertree with the maximum Wiener index among all $n$ - vertex and

$k$-uniform hypertrees, which is not isomorphic to any one of $\left\{E_{n, k}, B_{n, 2}^{3}, F_{n, 2}^{\prime}, F_{n, k}, P_{n, k}\right\}$, where $k \geq 2$.

Let $\Delta(T)$ be the maximum degree of $T$. Obviously, $\Delta(T) \geq 2$.

Suppose that $\Delta(T) \geq 5$. Then by Theorem 3.1, $T \cong B_{n, k}^{\Delta}$. Note that $B_{n, k}^{\Delta-1}$ is not isomorphic to any one of $\left\{E_{n, k}, B_{n, 2}^{3}, F_{n, 2}^{\prime}, F_{n, k}, P_{n, k}\right\}$ for $k \geq 2$. By Proposition 2.2, $W(T)=W\left(B_{n, k}^{\Delta}\right)<W\left(B_{n, k}^{\Delta-1}\right)$ for $k \geq 2$, a contradiction. Thus, $2 \leq \Delta(T) \leq 4$.

Suppose that $k=2$. Then $3 \leq \Delta(T) \leq 4$.

If $\Delta(T)=4$, by Theorem 3.1, then $T \cong B_{n, 2}^{4}$.

Suppose $\Delta(T)=3$. Note that $B_{n, 2}^{3}=F_{n, 2}$ and $T \neq F_{n, 2}^{\prime}$, $B_{n, 2}^{3}, P_{n, 2}$. If there are at least three vertices of degree 3 in $T$, then let $u, v$ be two vertices of degree 3 such that $d_{T}(u, v)$ 
is large as possible. Let $T_{1}, T_{2}, T_{3}$ be the vertex disjoint subhypergraphs of $T-v$ with $\bigcup_{i=1}^{3} V\left(T_{i}\right)=V(T) \backslash\{v\}$ such that $T\left[V\left(T_{i}\right) \cup\{v\}\right]$ is a 2 - uniform hypertrees for $1 \leq i \leq 3$. Suppose without loss of generality that $u \in V\left(T_{1}\right)$. Then $T\left[V\left(T_{2}\right) \cup\{v\}\right]$ and $T\left[V\left(T_{3}\right) \cup\{v\}\right]$ are two pendant paths at $v$. Let $l_{i}$ be the lengths of the pendant path $T\left[V\left(T_{i}\right) \cup\{v\}\right]$ at $v$, where $2 \leq i \leq 3$ and $l_{i} \geq 1$. Suppose $l_{2} \geq l_{3}$, then $T=G_{v}\left(l_{2}, l_{3}\right)$, where $G=T\left[V(T) \backslash V\left(T_{2}\right) \cup V\left(T_{3}\right)\right]$. Note $T^{\prime}=G_{v}\left(l_{2}+1, l_{3}-1\right) 2-$ uniform hypertrees with maximum degree 3 and $T \not F_{n, 2}^{\prime}, B_{n, 2}^{3}, P_{n, 2}$. By Proposition 2.2, $W\left(T^{\prime}\right)>W(T)$, which is a contradiction. So there are at most two vertices of degree 3 in $T$. If there is a unique vertex of degree 3 in $T$ and $T \neq F_{n, 2}^{\prime}, B_{n, 2}^{3}$, by Proposition $2.2, T$ is obtained by attaching a pendant edge at the fourth vertex of a path on $n-1$ vertices and $T \cong E_{n, 2}^{\prime}$. If there are exactly two vertices of degree 3 in $T, T$ is obtained by attaching two pendant edges at two internal vertices of a path on $n-2$ vertices, respectively, by Lemma $3.4, T \cong F_{n, 2}^{*}$. By direct calculation, we have $W\left(E_{n, 2}^{\prime}\right)>W\left(F_{n, 2}^{*}\right)$. Therefore, when $k=2, W(T) \leq W\left(B_{n, 2}^{4}\right)$ or $W(T) \leq W\left(E_{n, 2}^{\prime}\right)$, with equality if an only if $T \cong B_{n, 2}^{4}$ or $T \cong E_{n, 2}^{\prime}$.

Suppose that $k \geq 3$. Note that $2 \leq \Delta(T) \leq 4$

If $\Delta(T)=4$, by Theorem 3.1, then $T \cong B_{n, k}^{4}$. By Lemma 3.6, we have $W\left(B_{n, k}^{4}\right) \leq W\left(F_{n, k}^{\prime}\right)$.

If $\Delta(T)=3$, let $u$ be a vertex of degree 3. By similar argument of Theorem 3.1 in [4], $u$

must be the unique vertex of degree 3 in $T$. By Proposition 2.1, we see that $T$ is obtained

from a loose path $P_{n-(k-1), k}=\left(u_{0}, e_{1}, u_{1}, \cdots, e_{\frac{n-k}{k-1}}, u_{\frac{n-k}{k-1}}\right)$ by attaching an edge to a vertex of degree 2 of $P_{n-(k-1), k}$. From Proposition 2.2 and $T \nRightarrow B_{n, k}^{3}$, we have $T \cong F_{n, k}^{\prime}$.

Next we see the case of $\Delta(T)=2$.

Case 1. $\frac{n-1}{k-1}=8$. Since $T \notin E_{n, k}, F_{n, k}, P_{n, k}$, and $T$ has the maximum Wiener index, we get $T \cong H_{n, k}^{4}$. By direct calculation, we have $W\left(H_{n, k}^{4}\right) \leq W\left(F_{n, k}^{\prime}\right)$ for $k \geq 3$.

Case 2. $\frac{n-1}{k-1} \geq 9$. Note that $T \neq P_{n, k}$. Suppose that there are at least two edges such

that each edge has at least three vertices of degree 2 in $T$. Let $\mathrm{u}$ be a vertex of degree 1 in

$T$. Choose an edge $e=\left(w_{1}, \cdots, w_{k}\right)$ in $T$ with at lease three vertices of degree 2 such that

$d_{T}\left(u, w_{1}\right)$ is as large as possible, where $d_{T}\left(u, w_{1}\right)=d_{T}\left(u, w_{i}\right)-1$ for $2 \leq i \leq k$. Then there

are two pendant paths at different vertices of $e$, say $P$ at $w_{i}$ and $P$ at $w_{j}$, where $1 \leq i<j \leq k$. Let $p$ and $q$ be the lengths of $P$ and $Q$, respectively, where $p, q \geq 1$. Then

$T \cong H_{w_{i}, w_{j}}(p, q)$ with $\left.H=T\left[V(T) \backslash V(P \cup Q) \backslash\left\{w_{i}, w_{j}\right\}\right)\right]$.

Note that $d_{H}\left(w_{i}\right)=d_{H}\left(w_{j}\right)=1$. Without loss of generality, assume $p \geq q$. Note that $T^{\prime}=H_{w_{i}, w_{j}}(p+1, q-1)$ is a $k-$ uniform hypertree that is not isomorphic to $P_{n, k}$. If $T^{\prime}$ is also not isomorphic $F_{n, k}$ and $E_{n, k}$, then by Proposition 2.1, we have $W(T)<W\left(T^{\prime}\right)$, a contradiction. Thus, there is only one edge with at least three vertices of degree 2 in $T$ in this case. Next we will discuss the subcases of $T^{\prime} \cong F_{n, k}$ and $T^{\prime} \cong E_{n, k}$.

Subcase 2.1. $T^{\prime} \cong E_{n, k}$. Then $T$ is isomorphic to the $k-$ uniform hypertree obtained

From $\quad P_{n-2(k-1), k}=\left(u_{0}, e_{1}, u_{1}, \cdots, e_{\frac{n-1}{k-1}-2}, u_{\frac{n-1}{k-1}-2}\right)$

attaching a pendant $e^{\prime}$ at a vertex $w^{\prime}$

in $e_{2} \backslash\left\{u_{1}, u_{2}\right\}$ and attaching a pendant edge $e^{\prime \prime}$ at a vertex $w^{\prime \prime}$ in $e_{i} \backslash\left\{u_{i-1}, u_{i}\right\}$, where $3 \leq i \leq \frac{n-1}{k-1}-3$. Suppose without loss of generality that $T$ is such a hypertree.

If $4 \leq i \leq \frac{n-1}{k-1}-4$, for the hypertree $T$, by moving edge $e^{\prime}$ from $w^{\prime}$ to $u_{\frac{n-1}{k-1}-2}$ in $\frac{e_{n-1}-2}{k-1}$,

we get a $k$-uniform hypertree $T^{\prime \prime}$ and $T^{\prime \prime} \neq F_{n, k}, E_{n, k}$. Let $L$ be the unique path in $T$ from $w^{\prime}$ to $\frac{u_{n-1}}{k-1}-2$. Then

$$
\begin{gathered}
W_{T}\left(V(T) \backslash\left(e^{\prime} \backslash\left\{w^{\prime}\right\}\right)\right)=W_{T^{\prime \prime}}\left(V(T) \backslash\left(e^{\prime} \backslash\left\{w^{\prime}\right\}\right)\right), \\
W_{T}\left(e^{\prime} \backslash\left\{w^{\prime}\right\}\right)=W_{T^{\prime \prime}}\left(e^{\prime} \backslash\left\{w^{\prime}\right\}\right), \\
W_{T}\left(e^{\prime} \backslash\left\{w^{\prime}\right\}, V(L)\right)=W_{T^{\prime \prime}}\left(e^{\prime} \backslash\left\{w^{\prime}\right\}, V(L)\right), \\
W_{T}\left(e^{\prime} \backslash\left\{w^{\prime}\right\}, e^{\prime \prime} \backslash\left\{w^{\prime \prime}\right\}\right)-W_{T^{\prime \prime}}\left(e^{\prime} \backslash\left\{w^{\prime}\right\}, e^{\prime \prime} \backslash\left\{w^{\prime \prime}\right\}\right)=\left(2 i-\frac{n-1}{k-1}\right)(k-1)^{2},
\end{gathered}
$$




$$
W_{T}\left(e^{\prime} \backslash\left\{w^{\prime}\right\}, e_{1} \backslash\left\{u_{1}\right\}\right)-W_{T^{\prime \prime}}\left(e^{\prime} \backslash\left\{w^{\prime}\right\}, e_{1} \backslash\left\{u_{1}\right\}\right)=\left(4-\frac{n-1}{k-1}\right)(k-1)^{2} .
$$

Since

$$
\begin{gathered}
W(T)=W_{T}\left(V(T) \backslash\left(e^{\prime} \backslash\left\{\mathrm{w}^{\prime}\right\}\right)\right)+W_{T}\left(e^{\prime} \backslash\left\{w^{\prime}\right\}\right)+W_{T}\left(e^{\prime} \backslash\left\{w^{\prime}\right\}, V(L)\right) \\
+W_{T}\left(e^{\prime} \backslash\left\{\mathrm{w}^{\prime}\right\}, e^{\prime \prime} \backslash\left\{w^{\prime \prime}\right\}\right)+W_{T}\left(e^{\prime} \backslash\left\{w^{\prime}\right\}, e_{1} \backslash\left\{u_{1}\right\}\right), \\
W\left(T^{\prime \prime}\right)=W_{T^{\prime \prime}}\left(V(T) \backslash\left(e^{\prime} \backslash\left\{\mathrm{w}^{\prime}\right\}\right)\right)+W_{T^{\prime \prime}}\left(e^{\prime} \backslash\left\{w^{\prime}\right\}\right)+W_{T^{\prime \prime}}\left(e^{\prime} \backslash\left\{w^{\prime}\right\}, V(L)\right) \\
+W_{T^{\prime \prime}}\left(e^{\prime} \backslash\left\{\mathrm{w}^{\prime}\right\}, e^{\prime \prime} \backslash\left\{w^{\prime \prime}\right\}\right)+W_{T^{\prime \prime}}\left(e^{\prime} \backslash\left\{w^{\prime}\right\}, e_{1} \backslash\left\{u_{1}\right\}\right),
\end{gathered}
$$

We have

$$
\begin{aligned}
W_{T}(T)-W\left(T^{\prime \prime}\right) & =\left(2 i-\frac{n-1}{k-1}\right)(k-1)^{2}+\left(4-\frac{n-1}{k-1}\right)(k-1)^{2} \\
= & \left(4+2 i-2 \frac{n-1}{k-1}\right)(k-1)^{2}<0 .
\end{aligned}
$$

Thus, $\mathrm{W}(\mathrm{T})<\mathrm{W}\left(\mathrm{T}^{\prime \prime}\right)$, a contradiction.

If $i=\frac{n-1}{k-1}-3$ as $k \geq 3$, for the hypertree $T$, by moving edge $e_{1}$ from $\mathrm{u}_{1}$ to $u_{\frac{n-1}{k-1}-2}$ and moving $\mathrm{e}^{\prime}$ from $\mathrm{w}^{\prime}$ to $\mathrm{u}_{0} \in e_{1}$, then we get a $k$-uniform hypertree $T^{\prime \prime}$ and $T^{\prime \prime} \not F_{n, k}, E_{n, k}$. By the same calculation as above, $\mathrm{W}(\mathrm{T})<\mathrm{W}(\mathrm{T} ")$, a contradiction.

If $i=3$, in the hypertree $T$ by moving edge $e^{\prime}$ from $w^{\prime}$ to $u_{0}$ at $e_{1}$, then we get a $k$-uniform hypertree $T^{\prime \prime}$ and we see that $T^{\prime \prime} \cong H_{n, k}^{4}$. Through similar calculation, we get $\mathrm{W}(\mathrm{T})<\mathrm{W}\left(\mathrm{T}^{\prime \prime}\right)$, a contradiction.

Subcase 2.2. $T^{\prime} \cong E_{n, k}$. Then $T$ is isomorphic to the $k$ uniform hypertree obtained from

$$
P_{n-2(k-1), k}=\left(u_{0}, e_{1}, u_{1}, \cdots, e_{\frac{n-k}{k-1}-2}, u_{\frac{n-k}{k-1}-2}\right) \text { by attaching a }
$$

pendant $e^{\prime}$ at a vertex $w^{\prime}$ in $e_{3} \backslash\left\{u_{2}, u_{3}\right\}$ and attaching a pendant edge $e^{\prime \prime}$ at a vertex $\mathrm{w}^{\prime \prime}$ in $e_{i} \backslash\left\{u_{i-1}, u_{i}\right\}$, where $2 \leq i \leq \frac{n-1}{k-1}-3$ and $i \neq 3$. Suppose without loss of generality that $T$ is such a hypertree. By the same prove as above, we can get a contradiction. Thus $e$ is the unique edge with at least three vertices of degree 2.

Suppose that there are four vertices $w_{1}, w_{2}, w_{3}$ and $w_{4}$ of degree 2 in $e$ for $T$. Let $Q_{i}$ be the pendant path of length $l_{i}$ at $w_{i}$, where $l_{i} \geq 1$ for $i=1,2$. Suppose without loss of generality that $l_{1} \geq l_{2}$.

Let $\quad G=T\left[V(T) \backslash V\left(Q_{1} \cup Q_{2}\right) \backslash\left\{w_{1}, w_{2}\right\}\right] . \quad$ Then $T \cong G_{w_{1}, w_{2}}\left(l_{1}+1, l_{2}-1\right)$. Note that $d_{G}\left(w_{1}\right)=1$ and $T^{*} \cong G_{w_{1}, w_{2}}\left(l_{1}+1, l_{2}-1\right)$ is a $k$-uniform hypertree that is not isomorphic to $P_{n, k}$. If $T^{*}$ is also not isomorphic to $F_{n, k}$ and
$E_{n, k}$, by Proposition $2.1, \mathrm{~W}(\mathrm{~T})<\mathrm{W}\left(\mathrm{T}^{*}\right)$, a contradiction. Hence, there is only three vertices of degree 2 in the edge $e$.

If $T^{*} \cong F_{n, k}$, then $T$ is isomorphic to the $k$-uniform hypertree obtained from $P_{n-2(k-1), k}=\left(u_{0}, e_{1}, u_{1}, \cdots, e_{\frac{n-k}{k-1}-2}, u_{\frac{n-k}{k-1}-2}\right) \quad$ by attaching pendant edges $e^{\prime}$ and $e^{\prime \prime}$ at $y$ and $z$ in $e_{2} \backslash\left\{u_{1}, u_{2}\right\}$, respectively, and $y \neq z \quad . \quad T \cong H_{y, z}(1,1) \quad$ with $H=T\left[V(T) \backslash\left(e^{\prime} \cup e^{\prime \prime}\right) \backslash\{y, z\}\right]$. By moving $\frac{e_{\frac{n-1}{k-1}-2}}{\text { from }}$ $u_{\frac{n-1}{k-1}-3}$ to $u_{0}$ at $e_{1}$. Continue moving $e^{\prime}$ from $y$ to $\frac{u_{\frac{n-1}{k-1}-2} \text { at }}{k-1}$

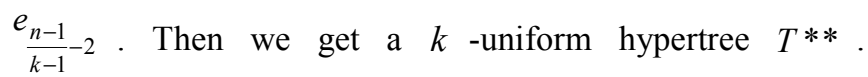
Obviously, $T^{* *} \cong H_{n, k}^{4}$, and by direct computation, we have $\mathrm{W}(\mathrm{T})<\mathrm{W}\left(\mathrm{T}^{* *}\right)$, a contradiction.

If $T^{*} \cong E_{n, k}$, , then $T$ is isomorphic to the $k$-uniform hypertree obtained from $P_{n-2(k-1), k}=\left(u_{0}, e_{1}, u_{1}, \cdots, e_{\frac{n-k}{k-1}-2}, u_{\frac{n-k}{k-1}-2}\right) \quad$ by attaching pendant edges $e^{\prime}$ and $e^{\prime \prime}$ at $y$ and $z$ in $e_{3} \backslash\left\{u_{2}, u_{3}\right\}$, respectively, $\quad y \neq z$. Note that $T \cong H_{y, z}(1,1)$ with $H=T\left[V(T) \backslash\left(e^{\prime} \cup e^{\prime \prime}\right) \backslash\{y, z\}\right]$. By $\mathrm{m} T^{* *} \cong H_{n, k}^{4}$ oving $e^{\prime}$ from $y$ to $u_{0}$ at $e_{1}$. Then we get a $k$-uniform hypertree $T^{* *}$. Obviously, and by Proposition 2.1, we have $\mathrm{W}(\mathrm{T})<\mathrm{W}\left(\mathrm{T}^{* *}\right)$, a contradiction.

As above, $T$ is a $k$-uniform hypertree obtained from $P_{n-(k-1), k}=\left(u_{0}, e_{1}, u_{1}, \cdots, e_{\frac{n-k}{k-1}-1}, u_{\frac{n-k}{k-1}-1}\right) \quad$ by attaching a pendant edge to a vertex of $e_{i} \backslash\left\{u_{i-1}, u_{i}\right\}$, with 
$4 \leq i \leq \frac{n-1}{k-1}-4$

If $\quad i=4 \quad$ or $\quad i=\frac{n-1}{k-1}-4, \quad$ then $\quad T \cong H_{n, k}^{4} \quad$. If $5 \leq i \leq \frac{n-1}{k-1}-5$, in the hypertree $T$, by moving the pendant

$$
V_{2}=V(T) \backslash\left(V\left(P^{\prime}\right) \cup e_{1} \cup e_{2} \cup e_{3} \cup e^{*}\right) .
$$

Then

$$
\begin{gathered}
W_{T}\left(V(T) \backslash\left(e^{*} \backslash\{w\}\right)\right)=W_{T^{*}}\left(V(T) \backslash\left(e^{*} \backslash\{w\}\right)\right), \\
\left.W_{T}\left(e^{*} \backslash\{w\}\right)=W_{T^{*}}\left(e^{*} \backslash\{w\}\right)\right), \\
W_{T}\left(e^{*} \backslash\{w\}, V\left(P^{\prime}\right)\right)=W_{T^{*}}\left(e^{*} \backslash\left\{w^{\prime}\right\}, V\left(P^{\prime}\right)\right), \\
W_{T}\left(e^{*} \backslash\left\{w^{\prime}\right\}, e_{1} \cup e_{2} \cup\left(e_{3} \backslash\left\{u_{3}\right\}\right)\right)=W_{T^{*}}\left(e^{*} \backslash\{w\}, e_{1} \cup e_{2} \cup\left(e_{3} \backslash\left\{u_{3}\right\}\right)\right)=3(i-4)(k-1)^{2}, \\
W_{T}\left(e^{*} \backslash\left\{w^{\prime}\right\}, V_{2}\right)-W_{T^{*}}\left(e^{*} \backslash\left\{w^{\prime}\right\}, V_{2}\right)=-(i-4)\left(\frac{n-k}{k-1}-i-1\right)(k-1)^{2} .
\end{gathered}
$$

Since

$$
\begin{aligned}
& W(T)=W_{T}\left(V(T) \backslash\left(e^{*} \backslash\{w\}\right)\right)+W_{T}\left(e^{*} \backslash\{w\}\right)+W_{T}\left(e^{*} \backslash\{w\}, V\left(P^{\prime}\right)\right) \\
& +W_{T}\left(e^{*} \backslash\left\{w^{\prime}\right\}, e_{1} \cup e_{2} \cup\left(e_{3} \backslash\left\{u_{3}\right\}\right)\right)+W_{T}\left(e^{*} \backslash\left\{w^{\prime}\right\}, V_{2}\right),
\end{aligned}
$$

and

$$
\begin{aligned}
& W\left(T^{*}\right)=W_{T^{*}}\left(V(T) \backslash\left(e^{*} \backslash\{w\}\right)\right)+W_{T^{*}}\left(e^{*} \backslash\{w\}\right)+W_{T^{*}}\left(e^{*} \backslash\{w\}, V\left(P^{\prime}\right)\right) \\
& +W_{T^{*}}\left(e^{*} \backslash\left\{w^{\prime}\right\}, e_{1} \cup e_{2} \cup\left(e_{3} \backslash\left\{u_{3}\right\}\right)\right)+W_{T^{*}}\left(e^{*} \backslash\left\{w^{\prime}\right\}, V_{2}\right),
\end{aligned}
$$

we have

$$
W(T)-W\left(T^{*}\right)=3(i-4)(k-1)^{2}-(\mathrm{i}-4)\left(\frac{\mathrm{n}-\mathrm{k}}{\mathrm{k}-1}-\mathrm{i}-1\right)(\mathrm{k}-1)^{2}=(\mathrm{i}-4)\left(4+\mathrm{i}-\frac{\mathrm{n}-1}{\mathrm{k}-1}\right)(\mathrm{k}-1)^{2}<0
$$

Thus, $\mathrm{W}(\mathrm{T})<\mathrm{W}\left(\mathrm{T}^{*}\right)$, a contradiction. So $T \cong H_{n, k}^{4}$. By direct calculation, we get $W\left(H_{n, k}^{4}\right) \leq W\left(F_{n, k}^{\prime}\right)$. as $k \geq 3$.

\section{Conclusion}

From above discussionthe, fifth Wiener index of hyperthree is obtained. $H_{n, k}^{i}$ be the $k$-uniform hypertree obtained from $P_{n-k+1, k}$ by attaching a pendant edge at a vertex in $e_{i} \backslash\left\{u_{i-1}, u_{i}\right\}$. Then $W\left(B_{n, k}^{3}\right) \geq W\left(H_{n, k}^{i}\right)$ with equality if and only if $i=3$ and $\frac{n-1}{k-1}=6$. For $k=2, n \geq 6$, $\Delta(T)=3 . \quad T$ be an any 2-uniform hypertree obtained by attaching two pendent edges at two internal vertices of a path on $n-2$ vertices, respectively. Then $W(T) \leq W\left(F_{n, 2}^{*}\right)$ with equality if and only if $T \cong F_{n, 2}^{*}$. For $\frac{n-1}{k-1} \geq 8$, let $T$ be an any $k$ - uniform hypertree on $n$ vertices. For $k=2$, $T \neq F_{n, 2}^{\prime} \quad, \quad B_{n, 2}^{3} \quad, \quad P_{n, 2} \quad, \quad$ then $W(T) \leq W\left(B_{n, 2}^{4}\right) \quad$ or $W(T) \leq W\left(E_{n, 2}^{\prime}\right)$, with equality if and only if $T \cong B_{n, 2}^{4}$ or $T \cong E_{n, 2}^{\prime}$.

For $k \geq 3, T \neq E_{n, k}, B_{n, 2}^{3}, F_{n, k}, P_{n, k}$, if $\frac{n-1}{k-1}=8$, then $W(T) \leq W\left(H_{n, k}^{4}\right)$ with equality if and only if $T \cong H_{n, k}^{4}$, and $W\left(H_{n, k}^{4}\right) \leq W\left(F_{n, k}^{\prime}\right)$. If $\frac{n-1}{k-1} \geq 9$, then $W(T) \leq W\left(F_{n, k}^{\prime}\right)$ with equality if and only if $T \cong F_{n, k}^{\prime}$. 


\section{Acknowledgements}

Supported by the NSFQH No. 2018-ZJ-925Q; NSFC No.11701311, QY201907; NSFGD No. 2016A030310307.

\section{References}

[1] F. R. K. Chung. The average distance and the indepence number. J. Graph Theory, 12: 229-235, 1988.

[2] I. Gutman, E. V. Konstantinova, V. A. Skorobogatov. Molecular hypergraphs and Clar structural formulas of benzenoid hydrocarbons. ACH Models Chem. 136 (1999) 539-548.

[3] A. A. Dobrynin, R. Entringer, I. Gutman. Wiener index of trees: theory and applications. Acta Appl. Math. 66 (2001) 211-249.

[4] H. Guo, B. Zhou, H. Lin, The Wiener Index of Uniform Hypergraphs, MATCH Commun. Math. Comput. Chem. 78 (2017) 133-152.

[5] E. V. Konstantinova, V. A. Skorobogatov. Application of hypergraph theory in chemistry. Discr. Math. 235 (2001) 365383.

[6] L. Sun, J. Wu, H. Cai, The Wiener Index of $r$-Uniform Hypergraphs, Bull. Malays. Math. 108 (2015) 43-52.

[7] Knor, M., Luzar, B., Škrekovski, R., Gutman, I.: On Wiener index of common neighborhood graphs. MATCH Commun. Math. Comput. Chem. 72, 321-332 (2014).

[8] H. Guo, B. Zhou, Properties of Degree Distance and Gutman Index of Uniform Hypergraphs, MATCH Commun. Math. Comput. Chem. 78 (2017) 213-220.

[9] Todeschini, R., Consonni, V.: Handbook of Molecular Descriptors. Wiley-VCH, Weinheim (2008).

[10] Wiener, H., Structual determination of paraffifin boiling points. J. Am. Chem. Soc. 69 (1), 17-20 (1947).

[11] H. Lin, B. Zhou, On the Wiener Index of Uniform Unicyclic Hypergraphs, MATCH Commun. Math. Comput. Chem. (2018).

[12] Xu, K., Liu, M., Das, K. C., Gutman, I., Furtula, B.: A survey on graphs extremal with respect to distance-based topological indices. MATCH Commun. Math. Comput. Chem. 71, 461508 (2014).
[13] J. K. Doyle, J. E. Graver. Mean distance in a graph. Discr. Math. 7 (1977) 147-154.

[14] P. J. Hansen, J. P. Jurs. Chemical applications of graph theory. Part I. Fundamentals and topological indices. J. Chem. Educ. 65 (1988) 574-580.

[15] H. Hosoya, Topological index. A newly proposed quantity characterizing the topological nature of structural of saturated hydrocarbons. Bull. Chem. Soc. Jpn. 44 (1971) 2332-2339.

[16] Lin, H.: Extremal Wiener index of trees with given number of vertices of even degree. MATCH Commun. Math. Comput. Chem. 72, 311-320 (2014).

[17] E. V. Konstantinova. Chemical Hypergraph theory. Pohang Univ. Sci. Tech, Pohang. 2001.

[18] H. Lin, B. Zhou. Distance spectral radius of uniform hypergraphs. Lin. Algebra Appl. 506 (2016) 564-578.

[19] J. Plesnik. On the sum of all distances in a graph or digraph. $J$. Graph Theory. 8 (1984) 1-21.

[20] D. H. Rouvray, Predicting chemistry from topology, Sci. Am. 255 (1986) 40-47.

[21] Hamzeh, A., Hossein-Zadeh, S., Ashrafifi, A. R.: Extremal graphs underWiener-type invariants. MATCH Commun. Math. Comput. Chem. 69, 47-54 (2013).

[22] Lin, H.: On the Wiener index of trees with given number of branching vertices. MATCH Commun. Math. Comput. Chem. 72, 301-310 (2014).

[23] Nadjafifi-Arani, M. J., Khodashenas, H., Ashrafifi, A. R.: A new method for computing Wiener index of dendrimer nanostars. MATCH Commun. Math. Comput. Chem. 69, 159164 (2013).

[24] Rodriguez, J. A.: On the Wiener index and the eccentric distance sum of hypergraphs. MATH Commun. Math. Comput. Chem. 54, 209-220 (2005).

[25] Škrekovski, R., Gutman, I.: Vertex version of the Wiener theorem. MATCH Commun. Math. Comput. Chem. 72, 295300 (2014).

[26] Tepavcevic, S., Wroble, A. T., Bissen, M., Wallace, D. J., Choi, Y., Hanley, L.: Photoemission studies of polythiophene and polyphenyl fifilms produced via surface polymerization by ion-assisted deposition. J. Phys. Chem. B. 109 (15), 71347140 (2005). 